This item was submitted to Loughborough's Research Repository by the author.

Items in Figshare are protected by copyright, with all rights reserved, unless otherwise indicated.

\title{
Synchrony in an array of integrate-and-fire neurons with dendritic structure
}

PLEASE CITE THE PUBLISHED VERSION

PUBLISHER

(C) American Physical Society

LICENCE

CC BY-NC-ND 4.0

\section{REPOSITORY RECORD}

Bressloff, P.C., and S. Coombes. 2019. "Synchrony in an Array of Integrate-and-fire Neurons with Dendritic Structure”. figshare. https://hdl.handle.net/2134/1721. 


\title{
Synchrony in an Array of Integrate-and-Fire Neurons with Dendritic Structure
}

\author{
P. C. Bressloff and S. Coombes \\ Nonlinear and Complex Systems Group, Department of Mathematical Sciences, Loughborough University, \\ Loughborough, Leics., LE11 3TU United Kingdom
}

(Received 21 January 1997)

\begin{abstract}
A one-dimensional array of pulse-coupled integrate-and-fire neurons, each filtering input through an idealized passive dendritic cable, is used to model the nonlinear behavior induced by axodendritic interactions in neural populations. The relative firing phase of the neurons in the array is derived in the weak-coupling regime. It is shown that for long-range excitatory coupling the phases can undergo a bifurcation from a synchronous state to a state of traveling oscillatory waves. We establish the possible role of dendritic structure in the desynchronization of cortical oscillations. [S0031-9007(97)03311-5]
\end{abstract}

PACS numbers: $87.10 .+\mathrm{e}, 05.45 .+\mathrm{b}$

In recent years neurobiologists have become increasingly interested in the role of the dendritic tree in neural behavior. Indeed, there is growing evidence to suggest that the dendrites constitute a highly complex, nonlinear information processing system (see, e.g., Ref. [1] and references therein). In spite of this, most mathematical studies of the dynamical behavior of neural populations neglect the influence of the dendritic tree completely. This is particularly surprising since, even at the passive level, the diffusive spread of activity along the dendritic tree implies that a neuron's response depends on (i) previous input history due to the existence of distributed delays and (ii) the particular locations of the stimulated synapses on the tree (i.e., the distribution of axodendritic connections). It is well known that delays can radically alter the dynamical behavior of a system. Moreover, the effects of distributed delays can differ considerably from those due to discrete delays arising, for example, from finite axonal transmission times [2]. Certain models do incorporate distributed delays using so-called $\alpha$ functions or some more general kernel [3]. However, the fact that these are not linked directly to dendritic structure means that feature (ii) has been neglected. As has recently been established within the context of neural pattern formation [4], the incorporation of dendritic structure in a neurodynamical model that combines features (i) and (ii) can lead to novel behavior.

In this Letter, we investigate the influence of dendritic structure on another important aspect of neurodynamics, namely, synchronization of coupled neural oscillators. There has been considerable work on the effects of delays on the synchronization of neural firing (see, e.g., Refs. [57]). In the case of sufficiently small axonal delays or fast synaptic responses, it is known that excitatory synaptic coupling tends to synchronize neural firing, whereas inhibitory coupling tends to generate antisynchrony. On the other hand, for larger axonal delays or slower synaptic responses, inhibition rather than excitation produces synchrony. It has also been shown that, in the case of a onedimensional array of coupled oscillators, destabilization of a synchronous state due to an increase in axonal delays can lead to traveling waves [8]. It has been suggested that this could account for the fact that oscillatory behavior in the visual cortex tends towards synchrony, whereas the olfactory cortex tends to produce traveling oscillatory waves; the latter has long-range excitatory connections and hence longer axonal delays. In this Letter, we shall show that the location of synaptic inputs on the dendritic tree also significantly affects the stability of synchronous oscillatory states and, hence, provides an alternative mechanism for the onset of traveling waves and other related phenomena.

For concreteness, consider a one-dimensional array of integrate-and-fire neurons distributed along the $x$ axis. Let $U(x, t)$ denote the somatic membrane potential of the neuron located at position $x \in \mathcal{R}$ at time $t$. Suppose that $U$ satisfies the equation

$$
\frac{\partial U(x, t)}{\partial t}=f(U(x, t))+I(x, t),
$$

where $I(x, t)$ is the net input into the soma due to synaptic connections with other neurons in the array. When $U(x)=1$ the neuron at $x$ fires and is reset to $U(x)=0$. In the absence of any synaptic input $I$, each neuron fires with a period $T=\int_{0}^{1} d U f(U)^{-1}$. The dendritic tree of a neuron is idealized as a uniform infinite onedimensional cable. Denote the membrane potential at the point $\xi \in \mathcal{R}$ on the cable of the neuron at $x$ by $V(\xi, x, t)$. Let $W\left(\xi,\left|x-x^{\prime}\right|\right)$ be the connection from a neuron at $x^{\prime}$ impinging on a synapse located at $\xi$ on the dendritic cable of a neuron at $x$. We shall assume for simplicity that there are no direct connections to the soma. Using standard cable theory [9], one can write the following equation for the dendritic potential $V$ :

$$
\begin{aligned}
\frac{\partial V}{\partial t}= & D \frac{\partial^{2} V}{\partial \xi^{2}}-\frac{V}{\tau}+I(\xi, x, t) \\
& +\int_{-\infty}^{\infty} W\left(\xi,\left|x-x^{\prime}\right|\right) E\left(x^{\prime}, t\right) d x^{\prime},
\end{aligned}
$$

where $E\left(x^{\prime}, t\right)$ represents the postsynaptic potential due to an input spike train from the neuron at $x^{\prime}, I(\xi, x, t)=$ $\rho(\xi)[U(x, t)-V(\xi, x, t)]$ is the current density flowing 
from the soma to the cable at $\xi$, and $\rho(\xi)$ is a conductance. We assume that the function $\rho(\xi)$ has compact support, i.e., it is localized to the contact region between the soma and the dendritic cable. By current conservation at the soma, $I(x, t)=-\int_{-\infty}^{\infty} I(\xi, x, t) d \xi$. In order to simplify our analysis, we shall assume that the current $I(\xi, x, t)$ in Eq. (2) is negligible compared to the synaptic current, and take $\rho(\xi)=\rho_{0} \delta(\xi)$, where $\delta$ is the Dirac delta function. Then $I(x, t)=\rho_{0} V(0, x, t)$ [after a term $-\rho_{0} U$ has been absorbed into the definition of the function $f$ in Eq. (1)]. We now solve Eq. (2) for $V(\xi, x, t)$ in terms of the synaptic inputs [4] and set $\xi=0$ to give

$$
\begin{aligned}
I(x, t)= & \rho_{0} \int_{-\infty}^{t} d t^{\prime} \int_{-\infty}^{\infty} d \xi^{\prime} G\left(\xi^{\prime}, t-t^{\prime}\right) \\
& \times \int_{-\infty}^{\infty} d x^{\prime} W\left(\xi^{\prime},\left|x-x^{\prime}\right|\right) E\left(x^{\prime}, t^{\prime}\right),
\end{aligned}
$$

where $G(\xi, t)=e^{-\epsilon_{0} t-\xi^{2} / 4 D t} / \sqrt{4 \pi D t}$ is the fundamental solution of the cable equation and $\epsilon_{0}=\tau^{-1}$. In the following analysis we fix length and time scales by setting $D=1$ and $\epsilon_{0}=1$. (Thus $\xi$ is measured in terms of electronic distance, which has typical values in the range $1-10 \mathrm{~cm}$. Typical values for the membrane time constant $\tau$ are 5-20 msec.)

In order to solve Eqs. (1) and (3) we shall assume that the coupling between neurons is weak so that, to a first approximation, each neuron still oscillates with period $T$. The relevant dynamical variable is then the phase $\phi(x)$ of each oscillator, and we can use a standard phase-reduction technique $[6,10,11]$. In particular, following Ref. [6], we introduce the nonlinear weak-coupling transform

$$
\phi+t / T \equiv \Psi(U)=T^{-1} \int_{0}^{U} \frac{d U^{\prime}}{f\left(U^{\prime}\right)} .
$$

The phase variable $\phi$ satisfies the equation

$$
\frac{\partial \phi(x, t)}{\partial t}=I(x, t) F(t / T+\phi(x, t)),
$$

where $F(z)=T^{-1} /\left[f \circ \Psi^{-1}(z)\right]$, and $F(z+n)=$ $F(z), n \in Z$. The neuron at $x$ fires when $t=[n-$ $\phi(x, t)] T$ for integer $n$. A synaptic input spike train then takes the form

$$
E(x, t)=\sum_{n=-\infty}^{0} E_{s}(t+[\phi(x, t)-n] T)
$$

for $0<t+T \phi(x, t)<T$, where $E_{s}$ represents the postsynaptic potential. For simplicity, we shall take $E_{s}(t)=$ $\delta(t)$, that is, we neglect the shape of the postsynaptic potential and the additional delays associated with the opening and closing of ionic channels; the latter could be incorporated using an $\alpha$ function $E_{s}(t)=\alpha^{2} t e^{-\alpha t}$ [6].

Now assume that $t / T$ varies much more quickly than $\phi(x)$. This is valid if the system is weakly coupled. Substituting Eqs. (6) and (3) into Eq. (5) and averaging over a single period $T$, one finds that

$$
\begin{aligned}
\frac{\partial \phi(x, t)}{\partial t}= & \rho_{0} \int_{-\infty}^{\infty} d \xi^{\prime} \int_{-\infty}^{\infty} d y W\left(\xi^{\prime},|y|\right) \\
& \times H\left(\xi^{\prime}, \phi(x+y, t)-\phi(x, t)\right),
\end{aligned}
$$

where

$$
H(\xi, \phi)=\int_{0}^{\infty} d \theta G(\xi, \theta T) F(\theta-\phi) .
$$

Equation (7) gives the general phase equation for a network of weakly coupled oscillators with dendritic structure. As discussed in [6], the function $F$ is the phase interaction function of the model in the case of an instantaneous synapse. For simplicity, we shall take $F(\theta)=-\sin 2 \pi \theta$, which is known to be a good approximation when $f$ of Eq. (1) has an experimentally determined form [12]. The function $H(\xi, \phi)$ involves the convolution of the instantaneous interaction function $F$ with the synaptic response function $G(\xi, t)$ which depends on the location $\xi$ of the synapse on the dendritic cable. Equation (8) may be evaluated using the following Fourier representation of the fundamental solution: $G(\xi, t)=$ $(2 \pi)^{-1} \int_{-\infty}^{\infty} d k e^{i k \xi-\epsilon(k) t}$, where $\epsilon(k)=k^{2}+1$. The result is

$$
\begin{aligned}
H(\xi, \phi)=\int_{-\infty}^{\infty} \frac{d k}{2 \pi} e^{i k \xi}[A(k) \sin 2 \pi \phi \\
-B(k) \cos 2 \pi \phi],
\end{aligned}
$$

where $\omega=2 \pi / T$,

$$
A(k)=\frac{\epsilon(k)}{\epsilon(k)^{2}+\omega^{2}}, \quad B(k)=\frac{\omega}{\epsilon(k)^{2}+\omega^{2}} .
$$

Following Crook et al. [8], we shall consider traveling wave solutions of equation Eq. (7) of the form $\bar{\phi}(x, t)=$ $\beta x+\Omega_{\beta} t$, with the frequency $\Omega_{\beta}$ satisfying the dispersion relation

$$
\Omega_{\beta}=\rho_{0} \int_{-\infty}^{\infty} d \xi^{\prime} \int_{-\infty}^{\infty} d y W\left(\xi^{\prime},|y|\right) H\left(\xi^{\prime}, \beta y\right) .
$$

It is clear that when $\beta=0$ the solution is synchronous. We wish to explore the stability of the traveling wave solutions $\bar{\phi}$. Linearizing Eq. (7) about $\bar{\phi}$ gives

$$
\begin{aligned}
\frac{\partial \psi(x, t)}{\partial t}= & \rho_{0} \int_{-\infty}^{\infty} d \xi^{\prime} \int_{-\infty}^{\infty} d y W\left(\xi^{\prime},|y|\right) H^{\prime}\left(\xi^{\prime}, \beta y\right) \\
& \times[\psi(x+y, t)-\psi(x, t)]
\end{aligned}
$$

which has solutions of the form $\psi(x, t)=e^{\lambda_{p} t+i p x}$ with

$$
\begin{aligned}
\lambda_{p}= & \rho_{0} \int_{-\infty}^{\infty} d \xi^{\prime} \int_{-\infty}^{\infty} d y W\left(\xi^{\prime},|y|\right) H^{\prime}\left(\xi^{\prime}, \beta y\right) \\
& \times\left[e^{i p y}-1\right] .
\end{aligned}
$$

Note that $H^{\prime}(\xi, \phi)$ indicates differentiation with respect to $\phi$. The traveling wave solution will be stable provided 
that $\operatorname{Re} \lambda_{p}<0$ for all $p \neq 0$. (The neutrally stable mode $\lambda_{0}=0$ represents constant phase shifts $\bar{\phi} \rightarrow \bar{\phi}+\delta$.)

We shall now study the stability of the synchronous state $\beta=0$ using Eqs. (9) and (13) for two general choices of the axodendritic weight distribution $W(\xi, x)$.

(I) Uncorrelated weight distribution. - Suppose that the weight distribution has the product form

$$
W(\xi, x)=P(\xi) W(x), \quad P(\xi) \geq 0,
$$

In other words, the distribution of axon collaterals across the dendritic tree of a postsynaptic neuron is independent of the separation between the neuron and the corresponding presynaptic neuron. The distribution $P(\xi)$ determines the probability density of these axon collaterals. Substituting Eq. (14) into (7) generates the standard phase equation describing weakly coupled oscillator systems [10]. The resulting phase interaction function is given by $H(\phi)=$ $\int_{-\infty}^{\infty} P(\xi) H(\xi, \phi) d \xi$. From equations Eqs. (9), (13), and (14), the real part of the eigenvalue $\lambda_{p}$ can be written as

$$
\begin{aligned}
\operatorname{Re} \lambda_{p}= & \frac{1}{2} \rho_{0} \int_{-\infty}^{\infty} d k \tilde{P}(k) A(k)[\tilde{W}(p+2 \pi \beta) \\
& +\tilde{W}(p-2 \pi \beta)-2 \tilde{W}(2 \pi \beta)],
\end{aligned}
$$

where $\tilde{P}(k)$ and $\tilde{W}(p)$ denote the Fourier transforms of $P(\xi)$ and $W(y)$. Following Ref. [8], suppose that the weight distribution $W(x)$ is excitatory and an exponentially decaying function of $y, W(y)=e^{-\gamma|y|} / 2$. The interactions are short range (long range) for large (small) $\gamma$. Then $\tilde{W}(p)=\gamma /\left(\gamma^{2}+p^{2}\right)$, and one can show that the term in square brackets of Eq. (15) is negative (positive) when $p^{2}>12 \pi^{2} \beta^{2}-\gamma^{2}\left(p^{2}<12 \pi^{2} \beta^{2}-\gamma^{2}\right)$. This means that the synchronous state $\beta=0$ will be stable (with $\gamma>0)$ if and only if $\bar{A} \equiv \int_{-\infty}^{\infty} d k \tilde{P}(k) A(k)$ is positive. To investigate the latter condition, set $P(\xi)=\delta\left(\xi-\xi_{0}\right)$ so that the location of each synaptic input on the dendritic tree is uniformly fixed at $\xi_{0}$. Then $\tilde{P}(k)=e^{i k \xi_{0}}$ and the integral $\bar{A}$ can be evaluated by closing the contour in the upper-half complex $k$ plane. One finds that $\bar{A}$ is positive if $\cos \left[r\left|\xi_{0}\right| \sin (\theta / 2)+\theta / 2\right]>0$ and is negative otherwise. Here $r^{2}=\sqrt{1+\omega^{2}}$ and $\theta=\tan ^{-1}(\omega)$ with $0 \leq \theta \leq \pi / 2$.

We deduce from the above analysis that, as the distance $\left|\xi_{0}\right|$ of the synapse from the soma increases from zero, it reaches a critical value $\xi_{0 c}= \pm(\pi-\theta) / 2 r \sin (\theta / 2)$. Increasing $\xi_{0}$ further produces alternating bands of stability and instability of the synchronous state as shown in Fig. 1. (These regions of stability/instability would be reversed in the case of inhibitory weights.) Here $\xi_{0}$ plays an analogous role to that of an axonal time delay, since it characterizes the effective delay due to diffusion along the dendrites [13]. It should be noted that, when the synchronous state is unstable, the solutions $\bar{\phi}$ for all $\beta \neq 0$ are also unstable. Hence, destabilization of the synchronous state does not lead to the formation of traveling oscillatory waves. It is also clear that for this example the range of the coupling $\gamma^{-1}$ does not influence the stability of the synchronous state. A model of two cou-

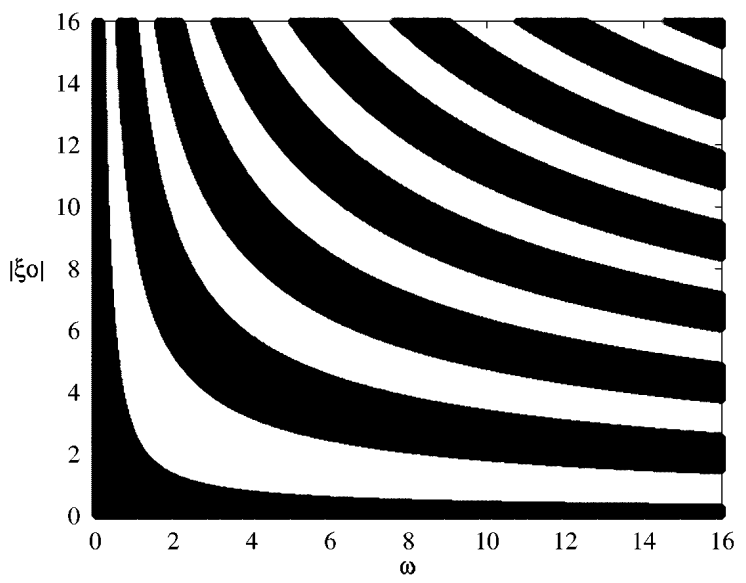

FIG. 1. Stability diagram for the uncorrelated weight distribution, $\left|\xi_{0}\right|$ vs $\omega$. White (black) regions correspond to instability (stability) for the synchronous solution.

pled somatic oscillators including passive dendritic structure has been independently explored by Crook [14] that captures the essential features of the uncorrelated weight distribution considered here.

(II) Correlated weight distribution. - When one considers the synaptic organization of the brain [15] one finds that the decoupling of network and dendritic coordinates is an over simplification. For example, recurrent collaterals of pyramidal cells in the olfactory cortex feed back onto basal dendrites of nearby cells and onto apical dendrites of distant pyramidal cells $[15,16]$. Thus a synapse tends to be located farther away from the soma as the separation between cortical neurons increases. This results in a reduction in the effectiveness of the synaptic connection due to diffusion along the dendritic tree. Motivated by this observation concerning the synaptic organization of cortical tissue, we make the following assumption about the distribution $W(\xi, x)$ : The average distance of a synapse from the soma $|\xi|$ increases with the separation $\left|x-x^{\prime}\right|$ between neurons. This property can be realized by a distribution of the form [2]

$$
W(\xi, x)=\frac{1}{2} W(x)[\delta(x-\xi)+\delta(x+\xi)] .
$$

For the weight distribution (16), our model has certain formal similarities to the model considered by Crook et al. [8]. Long-range interactions are delayed by the effects of diffusion in the former case [13] and by the effects of axonal transmission times in the latter case. Indeed, if one took $G(\xi, t)=\delta(t-|\xi| / \nu)$ then Eqs. (7), (8), and (16) would formally reduce to the model of Ref. [8] with $\nu$ interpreted as an axonal transmission speed.

Substituting Eq. (16) into Eq. (13) gives

$$
\begin{aligned}
\operatorname{Re} \lambda_{p}= & \frac{1}{2} \rho_{0} \int_{-\infty}^{\infty} d k A(k)[\tilde{W}(p+k+2 \pi \beta) \\
& +\tilde{W}(p+k-2 \pi \beta)-2 \tilde{W}(k+2 \pi \beta)] .
\end{aligned}
$$


In order to simplify the analysis, we shall take $W(x)=$ $W_{0} \Theta(L-|x|)$, where $\Theta$ is a step function. Here $L$ determines the range of the interactions rather than $\gamma^{-1}$ as in case I. Then $\tilde{W}(p)=2 W_{0} p^{-1} \sin p L$ and

$$
\begin{aligned}
\operatorname{Re} \lambda_{p}= & \frac{1}{2} \rho_{0} W_{0}[B(p+2 \pi \beta) \\
& +B(p-2 \pi \beta)-2 B(2 \pi \beta)],
\end{aligned}
$$

where $B(p)=2 \pi[A(p)+C(p)+C(-p)]$,

$$
C(p)=\frac{e^{-r L \cos \theta / 2}[p \sin \bar{\theta}-r \cos (\bar{\theta}+\theta / 2)]}{2 r\left[p^{2}+r^{2}+2 p r \sin (\theta / 2)\right]},
$$

and $\bar{\theta}=r L \sin (\theta / 2)+\theta / 2+p L$ with $r, \theta$ defined as in example I.

In the limit $L \rightarrow \infty$ (all-to-all coupling), $C(p) \rightarrow 0$ and one finds from Eq. (18) with $B(p)$ replaced by $A(p)$ that the synchronous state is unstable if $\omega>1$ and stable if $\omega<1$, where $\omega$ is the natural frequency of the integrateand-fire neurons. We also expect the synchronous state to be stable for sufficiently small values of $L$ for all $\omega$, since this corresponds to the limit of short-range interactions and hence small delays. We now use Eqs. (18) and (19) to determine the stability of traveling wave solutions with wave number $\beta$ as a function of the range of coupling $L$. We find that if $\omega<1$ then the synchronous state is stable for all $L$. On the other hand, if $\omega>1$ then the synchronous state is unstable for large $L$ and stable for small $L$. Whenever the synchronous state is unstable, there exist stable traveling wave solutions over a finite range of nonzero values of the wave number $\beta$. The stability region in the $(\beta, L)$ plane for $\omega=2$ is shown in Fig. 2. Note the nontrivial dependence of the dynamical behavior on the natural frequency $\omega$ of the oscillators. This should be contrasted with the analysis of Crook et al. who use a form for the phase interaction function $H$ that does not depend explicitly on either the properties of the dendrites or the natural frequency.

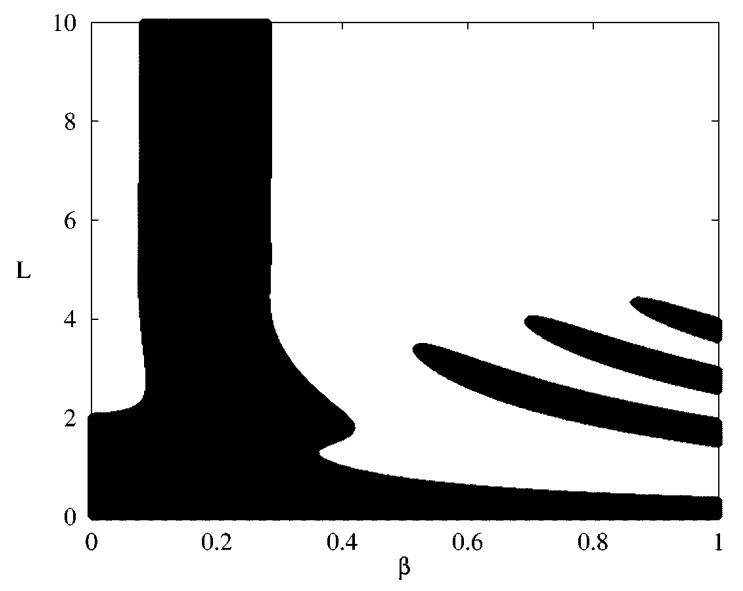

FIG. 2. Stability diagram for the correlated weight distribution, $L$ vs $\beta$ with $\omega=2$. White (black) regions correspond to unstable (stable) traveling wave solutions.
We conclude from our results that, for a correlated weight distribution, increasing the range of excitatory interactions can destabilize the synchronous state leading to the onset of stable traveling oscillatory waves when $\omega>1$. This can be confirmed numerically as will be shown in a more detailed analysis elsewhere. Thus dendritic structure provides an alternative to axonal delays [8] as a possible mechanism underlying the differences between oscillations in the visual and olfactory cortex. The fact that bifurcations occur when $\omega=O(1)$ and $L=$ $O(1)$ is particularly suggestive since these correspond to the typical frequencies $(10-100 \mathrm{~Hz})$ and length scales $(1-$ $10 \mathrm{~cm}$ ) relevant to cortical oscillations $[17,18]$.

This work was supported by Grant No. GR/K86220 from the EPSRC (UK).

[1] B. W. Mel, Neural Comput. 6, 1031 (1994).

[2] P. C. Bressloff, Phys. Rev. E 50, 2308 (1994).

[3] W. Gerstner, Phys. Rev. E 51, 738 (1995).

[4] P. C. Bressloff, Phys. Rev. Lett. 76, 4644 (1996).

[5] E. Niebur, H. H. Schuster, and D. M. Kammen, Phys. Rev. Lett. 67, 2753 (1991).

[6] C. V. Vreeswijk, L.F. Abbott, and G. B. Ermentrout, J. Comp. Neuro. 1, 313 (1994).

[7] S. Coombes and G. J. Lord, Phys. Rev. E 55, 2104 (1997).

[8] S. Crook, G. B. Ermentrout, and J.M. Bower (to be published).

[9] W. Rall, in Neural Theory and Modelling, edited by R. F. Reiss (Stanford University Press, Stanford, CA, 1964), pp. 73-97.

[10] G. B. Ermentrout and N. Kopell, SIAM J. Math. Anal. 15, 215 (1984).

[11] Y. Kuramoto, Chemical Oscillations, Waves, and Turbulence (Springer-Verlag, Berlin, 1984).

[12] D. Hansel, G. Mato, and C. Meunier, Europhys. Lett. 23, 367 (1993).

[13] When we speak of delays due to diffusion we are referring to the spreading out of input signals along the dendritic tree rather than the rate of transmission of these signals which is, of course, instantaneous. A useful definition is the "time to peak" $T\left(\xi_{0}\right)$ of an input at the soma due to a synaptic input at $\xi_{0} ; T\left(\xi_{0}\right)$ increases with $\left|\xi_{0}\right|$. We note that the time to peak can be as large as a few hundred msec, whereas axonal delays are typically $1-10 \mathrm{msec}$ (at least in myelinated fibers).

[14] S. M. Crook, Ph.D. thesis, University of Maryland, 1996 (unpublished).

[15] The Synaptic Organization of the Brain, edited by G. M. Shepherd (Oxford University Press, Oxford, 1990).

[16] M. A. Wilson and J. M. Bower, J. Neurophysiol. 67, 981 (1992).

[17] W. J. Freeman, Biol. Cybernet. 35, 21 (1979).

[18] C. M. Gray and W. Singer, Proc. Natl. Acad. Sci. U.S.A. 86, 1698 (1989). 